\title{
ON THE EXACTNESS OF CLASSICAL TRANSITION STATE THEORY FOR COLLINEAR COLLISIONS
}

\author{
Erik K. GRIMMELMANN and Lawrence L. LOHR Ir \\ Department of Chemistry, The University of Aichigan, \\ Ann Arbor, Michigan 48109, USA
}

Received 10 Tebruary 1977

\begin{abstract}
We have applied Pechukas and McLafferty'i test for the exactness of classic al transition state theory (TST) to the collinear subsurface of Porter and Karplus' $H_{3}$ hypersurface and have determined that $\Gamma S \Gamma$ is evact to at least $0.05928 \mathrm{~L} e \mathrm{~V}$ above the addle point energy.
\end{abstract}

\section{Introduction}

The basic assumption of classical transition state theory is that trajectories pass through the transition state at most once. Wigner [1] labeled this his "third assumption", and Miller [2] and Pechukas and McLafferty [3] have emphasized its importance more recently. Transition state theory (TST) is sand to be exact to energy $E_{0}$ if, and only if, for all $E \leqslant E_{0}$, no trajectory crosses the transition state more than once. Pechukas and McLafferty (designated hereafter as PM) have provided a test for determining if TST is exact to a given energy for a given potential energy surface. We have applied their test to the collinear subsurface of Porter and Karplus' [4] semi-conpincal $\mathrm{Ii}_{3}$ hypersurface * and have determined that TST is exact to at least $-4.291208 \mathrm{cV}$ (relative to $3 \mathrm{H}$ atoms), which is $0.059281 \mathrm{eV}$ above the saddle point energy. We report our results to six decimal places simply to indicate their numerical accuracy.

\section{Theory}

Pechukas and McLafferty's test is that TST is exact to energy $E$ if the band of the coordinate plane enclosed between the $V=E$ equipotentials can be covered by a continuous one-parameter family of straight-line segments such that at every point on a

F We have used their surface 2 . linc in the product (reactant) region the force $-\nabla V$ does not lic to the reactant (product) side of the line. If natural collision coordinates [5] are used, the lines of constant reaction coordinate provide a convenient continuous one-parameter family of straight-line segments so that the PM test follows as shown below from the equations of motion. The natural collision coordinates are $(s, v)$ and are expressed in mass werghted and skewed coordnates [6] in which the kinetic energy of motion is diagonal. The reaction coordinate $s$ is the distance along a generating curve $C$ and the local vibrationa! coordinate $v$ is the perpendicular distance from the curve $C$. The generating curve $\mathrm{C}$ divicles the courdindte plane unto two regions; $v$ is positive in one region and negative in the other. In this work we have taken $v$ as positive in the region containing dissociated configurations, that is, those in which the internuclear distances $R_{12}$ and $R_{23}$ are both large. Let $k(s)$ be the curvature of $C$ and let $\eta(s, v)=1+k(s) \cup$. The classical hamiltonian is

$H=\frac{1}{2}\left[\left(p_{s} / \eta\right)^{2}+p_{v}^{2}\right]+V$,

where $p_{s}$ and $p_{y}$ are the momenta conjugate to $s$ and $v$ respectively, and $V$ is the potential energy of the configuration $(s, v)$. The equations of motion are

$$
\begin{aligned}
& \mathrm{d} s / \mathrm{d} t=p_{s} / \eta^{2}, \\
& \mathrm{~d} v / \mathrm{d} t=p_{v}, \\
& \mathrm{~d} p_{s} / \mathrm{d} t=\left(v p_{s}^{2} / \eta^{3}\right) \mathrm{d} k / \mathrm{d} s-\partial V / \partial s, \\
& \mathrm{~d} p_{v} / \mathrm{d} t=\kappa p_{s}^{2} / \eta^{3}-\partial V / \partial v .
\end{aligned}
$$


The reaction coordinate $s$ can be considered a monotonic measure of reaction progress; it is ncgative in the reactant region, $z c$ ro at the transition state, and positive in the product region. If both $s$ and $p_{s}$ are positive, the trajectory is moving away from the transition state toward products. It follows from (2a) that as long as $p_{s}$ remains non-negative the trajectory can never return to the transition state. Using ( $2 \mathrm{c}$ ), Pechukas and McLafferty's test may be written as: TST is exact to energy $E$ if $\partial V / \partial s \leqslant 0$ for $s>0$ and $\partial V / \partial s \geqslant 0$ for $s<0$ for all points $(s, v)$ with $s \neq 0$ between the $V=E$ equipotentials. The converse, however, is not true; the PM tcst for exactness is sufficient but noi necessary. There may exist another continuous one-parameter family of straight-line segments for which the PM criterion for exactness is met. Even if no such family of line segments exists, TST may still be exact. The PM test only determines if there exists a trajectory which starts back toward the transition state; it says nothirg about whether such a trajectory ultimatcly returns to the transition state.

While in natural collision coordinates the lines of constant $s$ provide a continuous one-parameter family of straight-line segments suitable for the PM test, not every family of segments suitable for the test can be defined in such a manner. The use of natural collision coordinates in testing for exactness is a special casc of the more general PM test in that lines of constant reaction coordinate must not cross within the region between the $V=E$ equipotentials. Such crossings are, in themselves, insufficient to make a family of straightline segments unsuitable for use in the more general form of the PM test [3]. We now consider the conditions under which lines of constant reaction coordinate cross. Let $v_{*}(s)=-1 / k(s)$; the absolute value of $v_{*}(s)$ is the radius of curvature of the generating curve $C$ at $s$. At the points $\left(s, v_{*}(s)\right)$, lines of constant $s$ cross, $\eta\left(s, v_{*}(s)\right)=0$, and therefore the hamiltonian (1) and the equations of motion (2) are singular. When $\eta=0$ the jacobian of the transformation from $\left(Q_{1}, Q_{2}\right)$ to $(s, v)$ is also zero. Thus for a generating curve $\mathrm{C}$ to define natural collision coordinates suitable for use up to energy $E,\left(s, v_{*}(s)\right)$ must not lie within the region between the $V=E$ equipotentials for any $s$.

\section{Calculations}

In this work we have considered only collinear col- lisions of particles with equal masses on a symmetric potential energy surface, but one could consider collinear collisions of particles with arbitrary masses on asymmetric potential energy surfaces using similar methods. The coordinates $\left(Q_{1}, Q_{2}\right)$ in which the natural collision coordinates $(s, v)$ are expressed are mass weighted and skewed to diagonalize the kinctic energy of motion $T$,

$T=\frac{1}{2}\left[\left(\mathrm{~d} Q_{1} / \mathrm{d} t\right)^{2}+\left(\mathrm{d} Q_{2} / \mathrm{d} t\right)^{2}\right]$.

We have chosen $Q_{1}$ and $Q_{2}$ to be

$Q_{1}=c_{1}\left(R_{12}+R_{23}\right), \quad Q_{2}=c_{2}\left(R_{12}-R_{23}\right)$,

where $c_{1}$ and $c_{2}$ are functions of the particle masses and the units in which the $R$ 's and $Q$ 's are expressed. In this work we have expressed $Q_{1}$ and $Q_{2}$ in cgs units, $\mathrm{g}^{1 / 2} \mathrm{~cm}$. Since the potential energy surface used is symmetric about the line $R_{12}=R_{23}$, it is also symmetric about the line $Q_{2}=0$, and thus only one half of the surface need be examined for exactness. We have examined the half with $Q_{2} \geqslant 0$, which is the half with $s \geqslant 0$. The transition state, the path of stecpest ascent from the saddle point, is the line $Q_{2}=0$.

We have applied the PM test for exactness using several continuous one-parameter families of straightline segments: ca:h family is defined by a generating curve. First, the path of steepest descent from the saddle point was used as a generating curve for natural collision coordinates. The path was determined numerically by taking steps of $1 \times 10^{-23} \mathrm{~g}^{1 / 2} \mathrm{~cm}$. Halving the step size changed the path insignificantly. A cubic spline, fit to every 16th step, was used as the generating curve $C$. The curvature of $C$ is always non-positive and thus $v_{*}(s) \geqslant 0$ for all $s$. For fixed values of the reaction coordinate $s$, the vbrational coordinate $v$ was increased from 0 , where $\partial V / \partial s<0$, until either the root $\partial V / \partial s=0$ was located or $v=v_{*}(s)$ was reached. The location of the root was designated $v_{+}(s)$. Next, $v$ was decreased from 0 until a similar root was found and its location was designated $v_{-}(s)$. The curves $v_{-}(s), v_{+}(s)$, and $v_{*}(s)$ are shown in fig. 1 , and the potential energies along these curves, $V\left(v_{-}(s)\right), V\left(v_{+}(s)\right)$, and $V\left(v_{*}(s)\right)$, are shown in fig. 2 . If the energy of a trajectory $E$ is less than the lowest value of any of these potential energies, then $\partial V / \partial s \leqslant 0$ for the entire region between the $V=E$ equipotentials, and thus, by the PM test, TST is exact to energy $E$. The lowest value is on $V\left(v_{-}(s)\right)$ and is $-4.345827 \mathrm{eV}$, which is 0.004662 


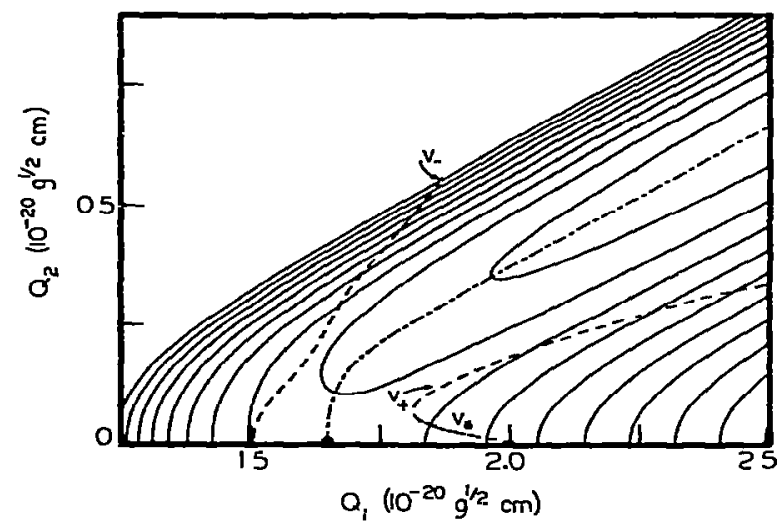

Iig 1. Lines of $z$ ero $a V / \partial s$, labeled as $v_{-}$and $v_{+}(--)$, for collinear $\mathrm{H}_{3}$. The reaction coordinate $s$ is the distance from the saddle point $(\bullet)$ along the path of steepest descent (--). Any trajectrry moving away from the transition state $\left(Q_{2}=0\right)$ cannot turn back toward it at any point within the region between $v_{-}$an $1 v_{+}$since $\partial V / \partial s<0$ within this region. Along $v_{*}(--), \eta=0$ and natural collision coordinates are undefıned. Successive equipotentrals (-) are separated by $0.2 \mathrm{eV}$; the lowest equipotential shown is $-4.6 \mathrm{eV}$.

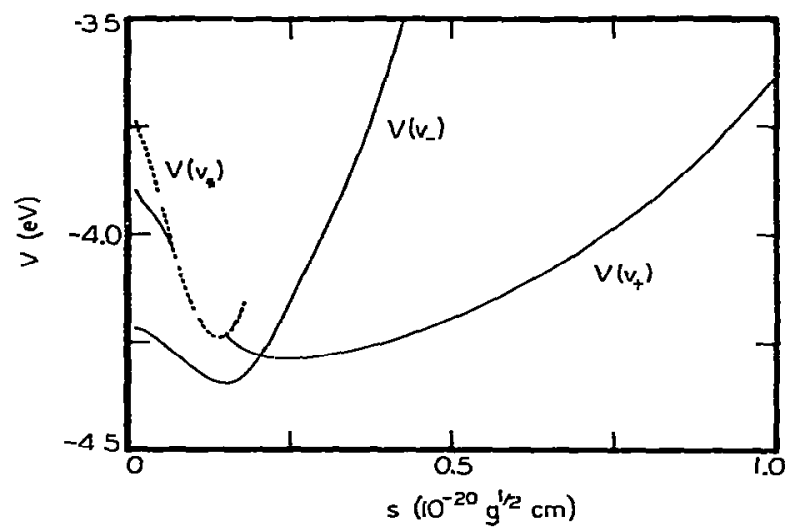

F-ig. 2. Potential energes along the lunes of zero $a V / \partial s$, labeled as $V\left(v_{-}\right)$and $V\left(v_{+}\right)(-)$. The potential energy $V\left(v_{*}\right)$ along the line of $\eta=0$ is also shown (-.-). See fig. 1 for the lines $v_{-} v_{+}$and $v_{*}$.

eV above the saddle point energy.

The second type of generating curve we have used consists of the equipotentials on the concave side of the path of steepest descent from the saddle point. Consider the region between the $V=l$ : equipotentials. For energies of interest this region lies entirely to the convex side of the generating curve, and thus the straight-line segments perpendicular to the generating curve cannot cross in this region. Use of this equipo- tential as the generating curve $\mathbf{C}$ trivially gives $\partial V / \partial s$ $=0$ when $v=0$. The sign of $\partial V / \partial s$ can be evaluated aiong the other $V=E$ equipotential, and if $\partial V / \partial s>0$ for any point along this other equipotential, then $E$ is above the energy to which the exactness of TST can be proven using the PM test with this choice of generating curve. For each $E$ a different generating curve of this type is required. Using this method we have found that $\partial V / \partial s$ becomes positive for at least one point when $E>-4.291208 \mathrm{eV}$. For this energy the sign of $\partial V / \partial s$ was calculated throughout the region between the $V=E$ equipotentials and found to be always negative. Thus, we have shown that TST is exact to at least $-4.291208 \mathrm{eV}$, which is $0.05928 \mathrm{I} \mathrm{cV}$ above the saddle point energy and higher than the value obtained using the path of steepest descent as the generating curve.

The third type of generating curve we have considered consists of the equipotentials on the convex side of the path of stecpest descent from the saddle point. At energies of interest some straight-line segments perpendicular to this gencrating curve cross within the region between the $V=E$ equipotentials, so that with these families of straight-line segments the more general form of the PM test must be uscd. Consider the region between the $V=F$ equipotentials. Along the equipotential used as the generating curve $C$, the force - $\nabla V$ trivially lies along the line segments perpendicular to $\mathrm{C}$. The direction of the force was calculated along the other equipotential and was found to lie to the side nearer the saddle point for at least one point when $E>-4.291208 \mathrm{eV}$, the same value (to seven significant figures) obtained using the other equiputential. For this energy the direction of the force was calculated throughout the region between the $V=E$ equipotentials and found always to lie to the transition state side of the straight-line segments.

Thus, if the region between the $V=-4.291208 \mathrm{eV}$ equipotentials is covered by either family of straightline segments which are perpendicular to one of these equipotentials, the force at every point on any line does not lie to the side of the line nearer the transition state. But if the region between the $V=-4.29[207 \mathrm{eV}$ equipotentials is covered by either family of segments defined in the same fashion, the force at some point on at least one line does lie to the side of the line nearer the transition state. Therefore, by applying the PM test, TST has been shown to be exact to at least 
$-4.291208 \mathrm{eV}$. The possibility remains that TST could be shown to be exact to a higher energy using a different type of family of straight-line segments.

\section{Discussion}

Using Pechukas and McLafferty's test, we have shown that for the collinear subsurface of Porter and Karplus' $\mathrm{H}_{3}$ hypersurface, transition state theory is exact to at least $0.059281 \mathrm{eV}$ above the saddle point energy. This is within the range of Pechukas and McLafferty's estimate that TST can be shown to be exact to an energy roughly between 0.01 and $0.1 \mathrm{eV}$ above the sacidle point energy. We have not shown, however, that trajectories of higher energy cannot return to the transition state once they have left it. Chapman ct al. [7] have shown that for the system considered here, TST is "essentially exact" for energies up to over $0.2 \mathrm{eV}$ above the saddle point energy. TST is said to be "cssentidly exact" for a given cnergy $E$ if at most only a small fraction of trajectories of energy $E$ pass through the transition state more than once.

The PM test for exactness was formulated for collinear triatomic collisions, but could be extended to more general collisions. Let $s$ be a reaction coordinate and let $\left\{v_{l}\right\}$ be the set of remaining coordinates (vibrations and rotations). Let each $v_{i}$ be orthogonal to $s$, but the various $v_{a}$ need not be orthogonal to one another $[8,9]$. As in the collinear case, let $s$ be negative in the reactant region, zero at the transition state, and positive in the product region. Transition state theory could be sand to be exact to energy $E$ if for all configurations $\left(s,\left\{v_{i}\right\}\right)$ with $s \neq 0$ for which $V\left(s,\left\{v_{i}\right\}\right)<E$, the conditions hold that $\partial V / \partial s \leqslant 0$ for $s>0$ and $\partial V / \partial s$ $\geqslant 0$ for $s<0$.

The information contained in fig. 2 has an addi- tional potential use. If one were calculating trajectories for a collinear triatomic system, and if only the probability of reaction and not the final distribution of energy between product modes were of interest, a diagram like fig. 2 would provide a criterion for stop. ping the integration of trajectories. For a given total energy, one can obtain from fig. 2 a value of the reaction coordinate $s$ beyond which the trajectory cannot return toward reactants and thus must continue to products. Once this value of $s$ is reached, integration may be stopped without fear that, were integration continued, the trajectory might lead back to reactants.

Finally it should be remembered that as useful a concept as a "reaction coordinate" is, there are many cases in which it is impossible to find a single reaction coordnate for which reaction "progress" is a monotonic function of reaction coordinate for all trajectories which lead from reactants to products.

\section{Acknowledgement}

We wish to thank The University of Michigan Computing Center for use of the Amdahl $470 \mathrm{~V} / 6$ computer.

\section{References}

[1] E. Wigner, Trans. Faraday Soc. 34 (1938) 29.

[2] W.Il. Miller, J. Chem. Phys. 61 (1974) 1823.

[3] P. Pechukas and F.J. McLafferty, J. Chem. Phys. 58 (1973) 1622.

[4] R.N. Porter and M. Karplus, J. Chem. Phys. 40 (1964) 1105.

[5] R.A. Marcus, J. Chem. Phys. 45 (1966) 4493, 4500.

[6] J.O. Hirschfelder, Intern. J. Quantum Chem. 3S (1969) 17.

[7] S. Chapman, S.M. Hornstein and W.H. Miller, J. Am. Chem. Soc. 97 (1975) 892.

[8] G.L. Hofacker, Z. Naturforsch. 18a (1963) 607.

[9] R.A. Marcus, J. Chem. Phys. 49 (1968) 2610, 2617. 